\title{
Active-Varying Sampling-Based Fault Detection Filter Design for Networked Control Systems
}

\author{
Yu-Long Wang, ${ }^{1}$ Tian-Bao Wang, ${ }^{2}$ and Wei-Wei Che ${ }^{3}$ \\ ${ }^{1}$ School of Electronics and Information, Jiangsu University of Science and Technology, Zhenjiang, Jiangsu 212003, China \\ ${ }^{2}$ School of Electrical and Information Engineering, Jiangsu University, Zhenjiang, Jiangsu 212013, China \\ ${ }^{3}$ Key Laboratory of Manufacturing Industrial Integrated Automation, Shenyang University, Shenyang, Liaoning 110044, China \\ Correspondence should be addressed to Yu-Long Wang; feixiangwyl@163.com
}

Received 17 March 2014; Revised 13 June 2014; Accepted 14 June 2014; Published 3 July 2014

Academic Editor: Masoud Hajarian

Copyright (C) 2014 Yu-Long Wang et al. This is an open access article distributed under the Creative Commons Attribution License, which permits unrestricted use, distribution, and reproduction in any medium, provided the original work is properly cited.

\begin{abstract}
This paper is concerned with fault detection filter design for continuous-time networked control systems considering packet dropouts and network-induced delays. The active-varying sampling period method is introduced to establish a new discretized model for the considered networked control systems. The mutually exclusive distribution characteristic of packet dropouts and network-induced delays is made full use of to derive less conservative fault detection filter design criteria. Compared with the fault detection filter design adopting a constant sampling period, the proposed active-varying sampling-based fault detection filter design can improve the sensitivity of the residual signal to faults and shorten the needed time for fault detection. The simulation results illustrate the merits and effectiveness of the proposed fault detection filter design.
\end{abstract}

\section{Introduction}

Networked control systems (NCSs) are spatially distributed systems whose sensors, actuators, and controllers are connected via a communication network. NCSs have been the subject of intensive research, and many interesting research topics have been reported, such as packet dropouts and network-induced delays [1-7], event-triggered control design [8-10], networked predictive control systems [11, 12], finitehorizon filtering $[13,14]$, quantization effects [14-16], output feedback control $[17,18]$, and nonuniformly distributed delays [19], to mention a few.

In NCSs, the sensor is assumed to sample at a fixed nominal period. However, computer loads, networks, and sporadic faults may cause sampling period jitter. Then, it is of paramount importance to study time-varying sampling periods $[20,21]$. It should be pointed out that the timevarying sampling periods considered in $[20,21]$ are induced by some external factors. Different from the time-varying sampling periods studied in $[20,21]$, the active-varying sampling periods were introduced in [22] to make full use of the network bandwidth.
For NCSs, the occurrence of faults is usually unavoidable. Then, it is interesting and important to study how to detect the occurrence of faults in time. Fault detection has been recognized as an important technique guaranteeing the safety and reliability of NCSs, and some nice results dealing with fault detection of NCSs have been reported [23, 24]. For example, Huang and Nguang [25] investigated robust fault estimation for NCSs with network-induced delays. Based on likelihood ratios for networked predictive control systems with random network-induced delays and clock asynchronism, Liu and Xia [26] proposed a fault detection and compensation scheme. Dong et al. [27] was concerned with the network-based robust fault detection problem for TakagiSugeno fuzzy systems with stochastic mixed time delays and successive packet dropouts.

Notice that the constant sampling period is considered in [23-27]. When dealing with fault detection for NCSs, if a constant sampling period $h$ is adopted and the network is occupied by the most users, $h$ should be large enough to avoid network congestion. Then, the network bandwidth cannot be sufficiently used when the network is idle. However, if the sensor can adjust the length of the sampling period actively 
to make full use of the network bandwidth, the needed time for fault detection may be shortened. It is interesting and important to study fault detection for NCSs adopting the active-varying sampling periods. For the problem of fault detection of NCSs, considering the active-varying sampling periods will introduce some difficulty for system modelling, and the active-varying sampling-based fault detection for NCSs has been paid no attention in the existing literature, which motivates the current study.

For discrete-time or discretized NCSs, suppose that $i_{k}$ is the sum of the number of consecutive packet dropouts and the length of network-induced delays, and $i_{m}$ and $i_{M}$ are known constants with $i_{m} \leq i_{k} \leq i_{M}, \bar{i}=\left\lfloor\left(i_{m}+i_{M}\right) / 2\right\rfloor$, where $\bar{i}$ is the largest integer smaller than or equal to $\left(i_{m}+\right.$ $\left.i_{M}\right) / 2$. Then one can conclude that at any instant $k, i_{m} \leq$ $i_{k} \leq \bar{i}$ or $\bar{i}<i_{k} \leq i_{M}$. On the other hand, for the specific instant $k$, the events $i_{m} \leq i_{k} \leq \bar{i}$ and $\bar{i}<i_{k} \leq i_{M}$ cannot occur simultaneously; such phenomenon is named as mutually exclusive distribution in this paper. When dealing with fault detection for discretized NCSs, how to make full use of the mutually exclusive distribution characteristic of packet dropouts and network-induced delays to derive less conservative results is of paramount importance, which is the second motivation of this paper.

This paper is concerned with fault detection filter (FDF) design for continuous-time NCSs considering packet dropouts and network-induced delays. By introducing the active-varying sampling period method, a new discretized model for fault detection NCSs is established. Based on the newly established model, the problem of fault detection filter design is studied. Even if the considered NCSs reduce to systems considering a constant sampling period, the proposed fault detection filter design is still applicable.

The contributions of this paper are summarized as follows.

(i) The active-varying sampling period method is introduced to establish a new discretized model for fault detection NCSs.

(ii) Fault detection filter design criteria for NCSs with packet dropouts and network-induced delays are derived. The derived design criteria can guarantee the sensitivity of the residual signal to faults.

(iii) The mutually exclusive distribution characteristic of packet dropouts and network-induced delays is made full use of to deal with fault detection of the considered NCSs, and the newly derived fault detection filter design criterion is proved to be less conservative.

The remainder of this paper is organized as follows. By introducing the active-varying sampling period method, Section 2 establishes a new discretized model for continuoustime NCSs with faults. Section 3 is concerned with the full order fault detection filter design. The simulation results are presented in Section 4. Conclusions are drawn in Section 5.

Notation. I and 0 represent an identity matrix and a zero matrix with appropriate dimensions, respectively. $*$ denotes the entries of a matrix implied by symmetry. Matrices, if not explicitly stated, are assumed to have appropriate dimensions.

\section{Modelling for Active-Varying Sampling-Based NCSs}

The continuous-time NCSs whose faults are to be detected are described by

$$
\begin{aligned}
& \dot{x}(t)=A x(t)+B u(t)+B_{\omega} \omega(t)+E_{f} f(t), \\
& y(t)=C x(t),
\end{aligned}
$$

where $x(t) \in \mathbb{R}^{n}, u(t) \in \mathbb{R}^{m}, y(t) \in \mathbb{R}^{s}, w(t) \in \mathbb{R}^{v}$, and $f(t) \in$ $\mathbb{R}^{q}$ are the state vector, control input vector, measurement output, disturbance input, and fault signal, respectively; $w(t)$ is assumed to belong to $L_{2}[0, \infty) ; A, B, B_{w}, E_{f}$, and $C$ are known constant matrices with appropriate dimensions.

In this paper, we assume that the system (1) is controlled through a one-channel network; packet dropouts and network-induced delays occur in the plant-to-FDF channel; the sensor is both clock-driven and event-driven, while the FDF and the actuator are event-driven. It should be pointed out that the results in this paper can be extended to deal with NCSs considering both the plant-to-FDF channel and the FDF-to-actuator channel.

When the network is idle and occupied by the most users, suppose that the plant-to-FDF network-induced delays are $d_{1}$ and $d_{2}$, respectively. If a constant sampling period is adopted and the network is occupied by the most users, one can choose $d_{2}$ as the sampling period to avoid network congestion. Suppose that $t_{k}$ is the latest sampling instant, $\tau_{k}$ is the network-induced delays of the measurement output $y_{k}$ $\left(y_{k}=C x_{k}\right.$ and $x_{k}$ is the plant state at the instant $\left.t_{k}\right)$, the instant that $y_{k}$ reaches the actuator is $\widetilde{k}$, and $h_{k}$ is the length of the $k$ th sampling period.

In the following, we will propose the active-varying sampling period method (see also [22] for a similar method) to improve the sensitivity of the residual signal to faults and shorten the needed time for fault detection.

Partitioning $\left[d_{1}, d_{2}\right]$ into $l$ equidistant small intervals $(l$ is a positive integer), then the next sampling instant (which is denoted as $\widehat{k}$ ) after $t_{k}$ can be chosen as

$$
\widehat{k}= \begin{cases}a_{1}, & \tilde{k}=a_{1}, \\ a_{2}, & \tilde{k} \in\left(a_{1}, a_{2}\right], \\ t_{k}+d_{2}, & \tilde{k} \geq t_{k}+d_{2},\end{cases}
$$

where $a_{1}=t_{k}+d_{1}+p\left(d_{2}-d_{1}\right) / l, a_{2}=t_{k}+d_{1}+(p+1)\left(d_{2}-d_{1}\right) / l$, $p=0,1, \ldots,(l-1)$. Then,

$$
h_{k}=d_{1}+\frac{b\left(d_{2}-d_{1}\right)}{l}, \quad b=0,1, \ldots, l ;
$$

that is, the sampling period $h_{k}$ switches in the finite set $9=$ $\left\{d_{1}, d_{1}+\left(d_{2}-d_{1}\right) / l, \ldots, d_{2}\right\}$. If $\tau_{k}>d_{2}$, the latest available measurement output will be used by the FDF, and $y_{k}$ will not be used even if it reaches the FDF eventually.

For a large enough $l$ and during the interval $\left[t_{k}, \widehat{k}\right)$, one can find that the measurement output adopted by the FDF is approximately $y_{k-i_{k}}$, where $i_{k}=i_{m}, i_{m}+1, \ldots$, and $i_{M}$, while $i_{M}-1$ is the maximum number of consecutive packet 
dropouts, $i_{m} \geq 0, i_{M}>0$, and $i_{m}<i_{M}$. Then the discretized representation of (1) can be described as

$$
\begin{gathered}
x_{k+1}=\Phi_{k} x_{k}+\Gamma_{1 k} u_{k}+\Gamma_{2 k} \omega_{k}+\Gamma_{3 k} f_{k}, \\
y_{k}=C x_{k},
\end{gathered}
$$

where $\Phi_{k}=e^{A h_{k}}, \Gamma_{1 k}=\int_{0}^{h_{k}} e^{A s} d s B, \Gamma_{2 k}=\int_{0}^{h_{k}} e^{A s} d s B_{\omega}$, and $\Gamma_{3 k}=\int_{0}^{h_{k}} e^{A s} d s E_{f}$.

At the $k$ th sampling instant, define the measurement output received by the FDF as $\bar{y}_{k}$. Then one has $\bar{y}_{k}=C x_{k-i_{k}}$. Based on the above statement, one can see that the fault detection filter can be described as

$$
\begin{gathered}
\widehat{x}_{k+1}=A_{f} \widehat{x}_{k}+B_{f} \bar{y}_{k}, \\
r_{k}=C_{f} \widehat{x}_{k},
\end{gathered}
$$

where $\widehat{x}_{k} \in \mathbb{R}^{n_{f}}$ and $r_{k} \in \mathbb{R}^{q}$ are the state of the fault detection filter and the residual signal, respectively; $A_{f}, B_{f}$, and $C_{f}$ are to be determined.

A reference residual model is usually needed to describe the desired behavior of the residual signal $r_{k}$. Introduce the following reference residual model, see also [28] for details:

$$
\begin{gathered}
\bar{x}_{k+1}=A_{W} \bar{x}_{k}+B_{W} f_{k}, \\
\bar{f}_{k}=C_{W} \bar{x}_{k}+D_{W} f_{k},
\end{gathered}
$$

where $\bar{x}_{k} \in \mathbb{R}^{n_{W}}$ and $\bar{f}_{k} \in \mathbb{R}^{q}$ are the state and the output of the reference residual model, respectively; $A_{W}, B_{W}, C_{W}$, and $D_{W}$ are known constant matrices of appropriate dimensions.

Define $\xi_{k}=\left[\begin{array}{lll}x_{k}^{T} & \bar{x}_{k}^{T} & \widehat{x}_{k}^{T}\end{array}\right]^{T}, v_{k}=\left[\begin{array}{lll}u_{k}^{T} & \omega_{k}^{T} & f_{k}^{T}\end{array}\right]^{T}$, and $e_{k}=$ $r_{k}-\bar{f}_{k}$. Then, one has

$$
\begin{gathered}
\xi_{k+1}=\phi_{1 \xi, k} \xi_{k}+\phi_{2 \xi} \xi_{k-i_{k}}+\phi_{3 \xi, k} \nu_{k}, \\
e_{k}=\phi_{1 e} \xi_{k}+\phi_{2 e} \nu_{k},
\end{gathered}
$$

where

$$
\begin{aligned}
& \phi_{1 \xi, k}=\left[\begin{array}{cc}
\widetilde{\Phi}_{k} & 0 \\
0 & A_{f}
\end{array}\right], \quad \phi_{2 \xi}=\left[\begin{array}{cc}
0 & 0 \\
B_{f} \widetilde{C} & 0
\end{array}\right], \\
& \phi_{3 \xi, k}=\left[\begin{array}{c}
\widetilde{\Gamma}_{k} \\
0
\end{array}\right], \quad \phi_{1 e}=\left[\begin{array}{ll}
\widetilde{C}_{W} & C_{f}
\end{array}\right] \text {, } \\
& \phi_{2 e}=\left[\begin{array}{lll}
0 & 0 & -D_{W}
\end{array}\right], \\
& \widetilde{\Phi}_{k}=\left[\begin{array}{cc}
\Phi_{k} & 0 \\
0 & A_{W}
\end{array}\right], \quad \widetilde{C}=\left[\begin{array}{ll}
C & 0
\end{array}\right], \\
& \widetilde{\Gamma}_{k}=\left[\begin{array}{ccc}
\Gamma_{1 k} & \Gamma_{2 k} & \Gamma_{3 k} \\
0 & 0 & B_{\omega}
\end{array}\right], \quad \widetilde{C}_{W}=\left[\begin{array}{ll}
0 & -C_{W}
\end{array}\right] .
\end{aligned}
$$

Remark 1. Generally speaking, the shorter the sampling period in NCSs, the better the system performance. However, a short sampling period will increase the possibility of network congestion. In the closed-loop system (7), the activevarying sampling period method is introduced to make full use of the network bandwidth, which will guarantee the system performance and avoid the occurrence of network congestion simultaneously.
If a constant sampling period $h$ is adopted, one can choose $h=d_{2}$ to avoid network congestion. In the case of adopting the constant sampling period $d_{2}$, the system in (7) reduces to

$$
\begin{gathered}
\xi_{k+1}=\phi_{1 \xi} \xi_{k}+\phi_{2 \xi} \xi_{k-i_{k}}+\phi_{3 \xi} \nu_{k} \\
e_{k}=\phi_{1 e} \xi_{k}+\phi_{2 e} \nu_{k},
\end{gathered}
$$

where $\phi_{1 \xi}=\left[\begin{array}{cc}\widetilde{\Phi} & 0 \\ 0 & A_{f}\end{array}\right], \phi_{3 \xi}=\left[\begin{array}{l}\widetilde{\Gamma} \\ 0\end{array}\right]$ with $\widetilde{\Phi}=\left[\begin{array}{cc}\Phi & 0 \\ 0 & A_{W}\end{array}\right], \widetilde{\Gamma}=$ $\left[\begin{array}{ccc}\Gamma_{1} & \Gamma_{2} & \Gamma_{3} \\ 0 & 0 & B_{\omega}\end{array}\right]$, and $\Phi=e^{A d_{2}}, \Gamma_{1}=\int_{0}^{d_{2}} e^{A s} d s B, \Gamma_{2}=\int_{0}^{d_{2}} e^{A s} d s B_{\omega}$, $\Gamma_{3}=\int_{0}^{d_{2}} e^{A s} d s E_{f}$, while $\phi_{2 \xi}, \phi_{1 e}$, and $\phi_{2 e}$ are the same as the corresponding items in (7).

To detect the occurrence of faults in time, one should construct a residual evaluation function. If the value of the residual evaluation function is larger than a given threshold, an alarm of faults will be generated. Define the residual evaluation function as

$$
\|r\|_{T} \triangleq \frac{1}{T} \sqrt{\sum_{k=t_{1}}^{t_{2}} r_{k}^{T} r_{k}}, \quad T=t_{2}-t_{1}+1 .
$$

Choose a threshold $J_{\text {th }}$ as follows:

$$
J_{\text {th }}=\sup _{v_{k} \in L_{2}, f_{k}=0}\|r\|_{T} .
$$

The fault detection logic is

$$
\begin{gathered}
\|r\|_{T}>J_{\text {th }}, \quad \text { with faults, } \\
\|r\|_{T} \leq J_{\text {th }}, \quad \text { without faults. }
\end{gathered}
$$

For the purpose of making full use of the mutually exclusive distribution characteristic of packet dropouts and networkinduced delays, one can introduce a scalar $\rho_{k}$, and

$$
\rho_{k}= \begin{cases}1, & i_{m} \leq i_{k} \leq \bar{i} \\ 0, & \bar{i}<i_{k} \leq i_{M}\end{cases}
$$

where $i_{m}, \bar{i}$, and $i_{M}$ are defined in Section 1 .

Based on the established model and the fault detection logic, this paper is concerned with the problem of fault detection filter design for NCSs considering the activevarying sampling periods. The mutually exclusive distribution characteristic of packet dropouts and network-induced delays is made full use of to derive a less conservative fault detection filter design criterion.

\section{Fault Detection Filter Design for Active-Varying Sampling-Based NCSs}

This section is concerned with fault detection filter design for NCSs considering the active-varying sampling periods. For this purpose, define $\Theta_{k}=\left[\begin{array}{llll}\xi_{k}^{T} & \xi_{k-1}^{T} & \cdots & \xi_{k-i_{M}}^{T}\end{array}\right]^{T}$, and choose the following Lyapunov functional:

$$
V_{k}\left(\Theta_{k}\right)=\sum_{j=1}^{4} V_{k j}\left(\Theta_{k}\right),
$$


where

$$
\begin{aligned}
& V_{k 1}\left(\Theta_{k}\right)=\xi_{k}^{T} P \xi_{k}, \\
& V_{k 2}\left(\Theta_{k}\right)=\sum_{j=k-i_{k}}^{k-1} \xi_{j}^{T} Q \xi_{j}+\sum_{\varrho=-i_{M}+1}^{-i_{m}} \sum_{j=k+\varrho}^{k-1} \xi_{j}^{T} Q \xi_{j}, \\
& V_{k 3}\left(\Theta_{k}\right)=\sum_{j=k-i_{m}}^{k-1} \xi_{j}^{T} R_{1} \xi_{j}+\sum_{j=k-i_{M}}^{k-1} \xi_{j}^{T} R_{2} \xi_{j}, \\
& V_{k 4}\left(\Theta_{k}\right)=\left(i_{M}-i_{m}\right) \sum_{\varrho=-i_{M}}^{-i_{m}-1} \sum_{j=k+\varrho}^{k-1} \eta_{j}^{T} Z \eta_{j},
\end{aligned}
$$

$P, Q, R_{1}, R_{2}$, and $Z$ are symmetric positive definite matrices, and $\eta_{j}=\xi_{j+1}-\xi_{j}$.

Then we state and establish the following result.

Theorem 2. For given scalars $i_{m} \geq 0, i_{M}>0$, and $\gamma>0$, if there exist symmetric positive definite matrices $P_{11}, P_{22}, Q_{11}$, $Q_{22}, R_{1,11}, R_{1,22}, R_{2,11}, R_{2,22}, Z_{11}$, and $Z_{22}$ and matrices $\widehat{A}, \widehat{B}$, $\widehat{C}, X_{1}, X_{2}, X_{3}, P_{12}, Q_{12}, R_{1,12}, R_{2,12}$, and $Z_{12}$, such that the following inequalities hold for $\rho_{k}=1$ or $\rho_{k}=0$ :

$$
\left[\begin{array}{cc}
\widetilde{\Omega} & \widetilde{\Pi}_{12} \\
* & \widetilde{\Pi}_{22}
\end{array}\right]<0,
$$

where

$$
\begin{aligned}
& \widetilde{\Omega}=\left[\begin{array}{ccccc}
\widetilde{\Omega}_{11} & 0 & 0 & 0 & 0 \\
* & \widetilde{\Omega}_{22} & \widetilde{\Omega}_{23} & 0 & 0 \\
* & * & \widetilde{\Omega}_{33} & \widetilde{\Omega}_{34} & 0 \\
* & * & * & \widetilde{\Omega}_{44} & 0 \\
* & * & * & * & -\gamma I
\end{array}\right], \\
& \widetilde{\Pi}_{12}=\left[\begin{array}{ccc}
H_{1} & H_{1}-\widetilde{G} & H_{4} \\
0 & 0 & 0 \\
H_{2} & H_{2} & 0 \\
0 & 0 & 0 \\
H_{3} & H_{3} & \phi_{2 e}^{T}
\end{array}\right], \\
& \widetilde{\Pi}_{22}=\operatorname{diag}\left\{\mathscr{X}_{1}, \mathscr{X}_{2},-\gamma I\right\}, \\
& \widetilde{\Omega}_{11}=-\widetilde{P}+\left(i_{M}-i_{m}+1\right) \widetilde{Q}+\widetilde{R}_{1}+\widetilde{R}_{2}, \\
& \widetilde{\Omega}_{22}=-\left(1-\rho_{k}\right) \widetilde{Q}-\widetilde{R}_{1}-\left(1+\rho_{k} \frac{i_{M}-\bar{i}}{\bar{i}-i_{m}}\right) \widetilde{Z}, \\
& \widetilde{\Omega}_{23}=\left(1+\rho_{k} \frac{i_{M}-\bar{i}}{\bar{i}-i_{m}}\right) \widetilde{Z}, \\
& \widetilde{\Omega}_{33}=-\widetilde{Q}-2 \widetilde{Z}-\left(\rho_{k} \frac{i_{M}-\bar{i}}{\bar{i}-i_{m}}+\left(1-\rho_{k}\right) \frac{\bar{i}-i_{m}}{i_{M}-\bar{i}}\right) \widetilde{Z}, \\
& \widetilde{\Omega}_{34}=\left(1+\left(1-\rho_{k}\right) \frac{\bar{i}-i_{m}}{i_{M}-\bar{i}}\right) \widetilde{Z},
\end{aligned}
$$

$$
\begin{aligned}
& \widetilde{\Omega}_{44}=-\widetilde{R}_{2}-\left(1+\left(1-\rho_{k}\right) \frac{\bar{i}-i_{m}}{i_{M}-\bar{i}}\right) \widetilde{Z}, \\
& H_{1}=\left[\begin{array}{cc}
\widetilde{\Phi}_{k}^{T} X_{1} & \widetilde{\Phi}_{k}^{T} X_{2} \\
\widehat{A} & \widehat{A}
\end{array}\right], \quad H_{2}=\left[\begin{array}{cc}
\widetilde{C}^{T} \widehat{B} & \widetilde{C}^{T} \widehat{B} \\
0 & 0
\end{array}\right], \\
& H_{3}=\left[\begin{array}{ll}
\widetilde{\Gamma}_{k}^{T} X_{1} & \widetilde{\Gamma}_{k}^{T} X_{2}
\end{array}\right], \quad H_{4}=\left[\begin{array}{c}
\widetilde{C}_{W}^{T} \\
\widehat{C}
\end{array}\right], \\
& \mathscr{X}_{1}=\widetilde{P}-\widetilde{G}-\widetilde{G}^{T}, \\
& \mathscr{X}_{2}=\left(i_{M}-i_{m}\right)^{-2}\left(\widetilde{Z}-\widetilde{G}-\widetilde{G}^{T}\right) \text {, } \\
& \widetilde{P}=\left[\begin{array}{cc}
P_{11} & P_{12} \\
* & P_{22}
\end{array}\right], \quad \widetilde{Q}=\left[\begin{array}{cc}
Q_{11} & Q_{12} \\
* & Q_{22}
\end{array}\right], \\
& \widetilde{R}_{1}=\left[\begin{array}{cc}
R_{1,11} & R_{1,12} \\
* & R_{1,22}
\end{array}\right], \quad \widetilde{R}_{2}=\left[\begin{array}{cc}
R_{2,11} & R_{2,12} \\
* & R_{2,22}
\end{array}\right], \\
& \widetilde{Z}=\left[\begin{array}{cc}
Z_{11} & Z_{12} \\
* & Z_{22}
\end{array}\right], \quad \widetilde{G}=\left[\begin{array}{cc}
X_{1} & X_{2} \\
X_{3} & X_{3}
\end{array}\right],
\end{aligned}
$$

then under the fault detection filter (5) with

$$
\begin{aligned}
A_{f} & =G_{3}^{-T} \widehat{A}^{T} G_{3}^{-1} G_{4}, \\
B_{f} & =G_{3}^{-T} \widehat{B}^{T}, \\
C_{f} & =\widehat{C}^{T} G_{3}^{-1} G_{4},
\end{aligned}
$$

the residual system (7) is asymptotically stable with an $H_{\infty}$ norm bound $\gamma$.

Proof. Taking the time difference of the Lyapunov functional $V_{k}\left(\Theta_{k}\right)$ in (14) along the trajectory of the system (7), one has

$$
\begin{aligned}
\Delta V_{k}\left(\Theta_{k}\right) & =V_{k+1}\left(\Theta_{k+1}\right)-V_{k}\left(\Theta_{k}\right) \\
& =\sum_{j=1}^{4} \Delta V_{k j}\left(\Theta_{k}\right),
\end{aligned}
$$

where

$$
\begin{aligned}
\Delta V_{k 1}\left(\Theta_{k}\right) & =\xi_{k+1}^{T} P \xi_{k+1}-\xi_{k}^{T} P \xi_{k}, \\
\Delta V_{k 2}\left(\Theta_{k}\right)= & \left(i_{M}-i_{m}+1\right) \xi_{k}^{T} Q \xi_{k} \\
& +\sum_{j=k-i_{m}+1}^{k-1} \xi_{j}^{T} Q \xi_{j}-\sum_{j=k-i_{k}+1}^{k-1} \xi_{j}^{T} Q \xi_{j} \\
& +\sum_{j=k-i_{k+1}+1}^{k-i_{m}} \xi_{j}^{T} Q \xi_{j} \\
& -\sum_{j=k-i_{M}+1}^{k-i_{m}} \xi_{j}^{T} Q \xi_{j}-\xi_{k-i_{k}}^{T} Q \xi_{k-i_{k}} .
\end{aligned}
$$


Notice that $\sum_{j=k-i_{k+1}+1}^{k-i_{m}} \xi_{j}^{T} Q \xi_{j}-\sum_{j=k-i_{M}+1}^{k-i_{m}} \xi_{j}^{T} Q \xi_{j} \leq 0$. Considering the mutually exclusive distribution characteristic of $i_{k}$, one has

$$
\sum_{j=k-i_{m}+1}^{k-1} \xi_{j}^{T} Q \xi_{j}-\sum_{j=k-i_{k}+1}^{k-1} \xi_{j}^{T} Q \xi_{j} \leq-\left(1-\rho_{k}\right) \xi_{k-i_{m}}^{T} Q \xi_{k-i_{m}} .
$$

Then,

$$
\begin{gathered}
\Delta V_{k 2}\left(\Theta_{k}\right) \leq\left(i_{M}-i_{m}+1\right) \xi_{k}^{T} Q \xi_{k}-\xi_{k-i_{k}}^{T} Q \xi_{k-i_{k}} \\
-\left(1-\rho_{k}\right) \xi_{k-i_{m}}^{T} Q \xi_{k-i_{m}}, \\
\Delta V_{k 3}\left(\Theta_{k}\right)=\xi_{k}^{T}\left(R_{1}+R_{2}\right) \xi_{k}-\xi_{k-i_{m}}^{T} R_{1} \xi_{k-i_{m}}-\xi_{k-i_{M}}^{T} R_{2} \xi_{k-i_{M}} .
\end{gathered}
$$

Adopting the mutually exclusive distribution characteristic of $i_{k}$ and the Jensen integral inequality in [29], one has

$$
\begin{aligned}
\Delta V_{k 4}\left(\Theta_{k}\right)= & \left(i_{M}-i_{m}\right)^{2}\left(\xi_{k+1}-\xi_{k}\right)^{T} Z\left(\xi_{k+1}-\xi_{k}\right) \\
& -\left(i_{M}-i_{m}\right) \sum_{j=k-i_{M}}^{k-i_{m}-1} \eta_{j}^{T} Z \eta_{j} \\
\leq & \left(i_{M}-i_{m}\right)^{2}\left(\xi_{k+1}-\xi_{k}\right)^{T} Z\left(\xi_{k+1}-\xi_{k}\right) \\
& -\rho_{k} \frac{i_{M}-\bar{i}}{\bar{i}-i_{m}} \varphi_{1 k}^{T} Z \varphi_{1 k} \\
& -\varphi_{1 k}^{T} Z \varphi_{1 k}-\varphi_{2 k}^{T} Z \varphi_{2 k} \\
& -\left(1-\rho_{k}\right) \frac{\bar{i}-i_{m}}{i_{M}-\bar{i}} \varphi_{2 k}^{T} Z \varphi_{2 k}
\end{aligned}
$$

and $\varphi_{1 k}=\xi_{k-i_{m}}-\xi_{k-i_{k}}, \varphi_{2 k}=\xi_{k-i_{k}}-\xi_{k-i_{M}}$.

Then, by combining (7) and (20)-(23) together, one has

$$
\Delta V_{k}\left(\Theta_{k}\right)+\gamma^{-1} e_{k}^{T} e_{k}-\gamma v_{k}^{T} \nu_{k} \leq \tilde{\xi}_{k}^{T}(\Omega+\Xi) \tilde{\xi}_{k},
$$

where $\widetilde{\xi}_{k}=\left[\begin{array}{lllll}\xi_{k}^{T} & \xi_{k-i_{m}}^{T} & \xi_{k-i_{k}}^{T} & \xi_{k-i_{M}}^{T} & v_{k}^{T}\end{array}\right]^{T}$ and

$$
\Omega=\left[\begin{array}{ccccc}
\Omega_{11} & 0 & 0 & 0 & 0 \\
* & \Omega_{22} & \Omega_{23} & 0 & 0 \\
* & * & \Omega_{33} & \Omega_{34} & 0 \\
* & * & * & \Omega_{44} & 0 \\
* & * & * & * & -\gamma I
\end{array}\right]
$$

with

$$
\begin{aligned}
& \Omega_{11}=-P+\left(i_{M}-i_{m}+1\right) Q+R_{1}+R_{2}, \\
& \Omega_{22}=-\left(1-\rho_{k}\right) Q-R_{1}-\left(1+\rho_{k} \frac{i_{M}-\bar{i}}{\bar{i}-i_{m}}\right) Z, \\
& \Omega_{23}=\left(1+\rho_{k} \frac{i_{M}-\bar{i}}{\bar{i}-i_{m}}\right) Z,
\end{aligned}
$$

$$
\begin{aligned}
& \Omega_{33}=-Q-2 Z-\left(\rho_{k} \frac{i_{M}-\bar{i}}{\bar{i}-i_{m}}+\left(1-\rho_{k}\right) \frac{\bar{i}-i_{m}}{i_{M}-\bar{i}}\right) Z, \\
& \Omega_{34}=\left(1+\left(1-\rho_{k}\right) \frac{\bar{i}-i_{m}}{i_{M}-\bar{i}}\right) Z \text {, } \\
& \Omega_{44}=-R_{2}-\left(1+\left(1-\rho_{k}\right) \frac{\bar{i}-i_{m}}{i_{M}-\bar{i}}\right) Z \text {, } \\
& \Xi=\Upsilon_{1}^{T} P \Upsilon_{1}+\left(i_{M}-i_{m}\right)^{2} \Upsilon_{2}^{T} Z \Upsilon_{2}+\gamma^{-1} \Upsilon_{3}^{T} \Upsilon_{3}, \\
& \Upsilon_{1}=\left[\begin{array}{lllll}
\phi_{1 \xi, k} & 0 & \phi_{2 \xi} & 0 & \phi_{3 \xi, k}
\end{array}\right] \\
& \Upsilon_{2}=\left[\begin{array}{lllll}
\left(\phi_{1 \xi, k}-I\right) & 0 & \phi_{2 \xi} & 0 & \phi_{3 \xi, k}
\end{array}\right], \\
& \Upsilon_{3}=\left[\begin{array}{lllll}
\phi_{1 e} & 0 & 0 & 0 & \phi_{2 e}
\end{array}\right] .
\end{aligned}
$$

From (24), one can see that if $\Omega+\Xi<0$, then $\Delta V_{k}\left(\Theta_{k}\right)+$ $\gamma^{-1} e_{k}^{T} e_{k}-\gamma \nu_{k}^{T} \nu_{k}<0$. By using Schur complement, $\Omega+\Xi<0$ is equivalent to

$$
\left[\begin{array}{ll}
\Omega & \Pi_{12} \\
* & \Pi_{22}
\end{array}\right]<0,
$$

where $\Pi_{12}=\left[\begin{array}{lll}\Upsilon_{1}^{T} & \Upsilon_{2}^{T} & \Upsilon_{3}^{T}\end{array}\right]$ and $\Pi_{22}=\operatorname{diag}\left\{-P^{-1}\right.$, $\left.-\left(i_{M}-i_{m}\right)^{-2} Z^{-1},-\gamma I\right\}$.

Introduce a matrix $G=\left[\begin{array}{ll}G_{1} & G_{2} \\ G_{3} & G_{4}\end{array}\right]$, where the selection of $G_{3}$ and $G_{4}$ is discussed in Remark 3. Pre- and postmultiplying both sides of (27) with $\operatorname{diag}\{\underbrace{I, \ldots, I}_{5}, G^{T}, G^{T}, I\}$ and its transpose, and considering that $Z-G-G^{T} \geq-G^{T} Z^{-1} G$ and $P-G-G^{T} \geq-G^{T} P^{-1} G$, one can see that if the inequalities in (28) are satisfied for $\rho_{k}=1$ or $\rho_{k}=0$, the inequalities in (27) are also satisfied as follows:

$$
\left[\begin{array}{ll}
\Omega & \bar{\Pi}_{12} \\
* & \bar{\Pi}_{22}
\end{array}\right]<0
$$

where

$$
\bar{\Pi}_{12}=\left[\begin{array}{lll}
\Upsilon_{1}^{T} G & \Upsilon_{2}^{T} G & \Upsilon_{3}^{T}
\end{array}\right]
$$

$$
\begin{aligned}
& \bar{\Pi}_{22} \\
& \quad=\operatorname{diag}\left\{P-G-G^{T},\left(i_{M}-i_{m}\right)^{-2}\left(Z-G-G^{T}\right),-\gamma I\right\} .
\end{aligned}
$$

Suppose that $M=\operatorname{diag}\left\{I, G_{4}^{-1} G_{3}\right\}$. Pre- and postmultiplying both sides of (28) with $\operatorname{diag}\{\underbrace{M^{T}, \ldots, M^{T}}, I, M^{T}, M^{T}, I\}$ and its transpose and defining $M^{T} G M=\widetilde{G}=\left[\begin{array}{ll}X_{1} & X_{2} \\ X_{3} & X_{3}\end{array}\right], G_{1}=X_{1}$, $G_{2} G_{4}^{-1} G_{3}=X_{2}, G_{3}^{T} G_{4}^{-T} G_{3}=X_{3}, M^{T} P M=\widetilde{P}=\left[\begin{array}{cc}P_{11} & P_{12} \\ * & P_{22}\end{array}\right]$, $M^{T} \mathrm{Q} M=\widetilde{Q}=\left[\begin{array}{cc}\mathrm{Q}_{11} & \mathrm{Q}_{12} \\ * & \mathrm{Q}_{22}\end{array}\right], M^{T} R_{1} M=\widetilde{R}_{1}=\left[\begin{array}{cc}R_{1,11} & R_{1,12} \\ * & R_{1,22}\end{array}\right]$, $M^{T} R_{2} M=\widetilde{R}_{2}=\left[\begin{array}{cc}R_{2,11} & R_{2,12} \\ * & R_{2,22}\end{array}\right], M^{T} Z M=\widetilde{Z}=\left[\begin{array}{cc}Z_{11} & Z_{12} \\ * & Z_{22}\end{array}\right]$, $G_{3}^{T} G_{4}^{-T} A_{f}^{T} G_{3}=\widehat{A}, B_{f}^{T} G_{3}=\widehat{B}, G_{3}^{T} G_{4}^{-T} C_{f}^{T}=\widehat{C}$, one can see that the inequalities in (28) are equivalent to the ones in (16). 
Then, by using the definition of $H_{\infty}$ performance, one can see that if the inequalities in (16) are satisfied for $\rho_{k}=1$ or $\rho_{k}=0$, the system (7) is asymptotically stable with an $H_{\infty}$ norm bound $\gamma$. This completes the proof.

Remark 3. Notice that the feasibility of the inequalities in (16) implies the nonsingularity of $\widetilde{G}$ and $X_{3}$. Since $X_{3}=G_{3}^{T} G_{4}^{-T} G_{3}$, the nonsingularity of $X_{3}$ implies that $G_{3}$ and $G_{4}$ are also nonsingular. By using the singular value decomposition of $X_{3}^{T}$, one can obtain the matrices $G_{3}^{T}$ and $G_{4}^{-1} G_{3}$.

It should be pointed out that the active-varying sampling period method is adopted in Theorem 2 to deal with fault detection filter design. Even if the constant sampling period is adopted, the fault detection filter design in Theorem 2 is still applicable. For the purpose of comparison, we establish the following fault detection filter design criterion for the system (9) with a constant sampling period.

Corollary 4. For given scalars $i_{m} \geq 0, i_{M}>0$, and $\gamma>0$, if there exist symmetric positive definite matrices $P_{11}, P_{22}, Q_{11}$, $Q_{22}, R_{1,11}, R_{1,22}, R_{2,11}, R_{2,22}, Z_{11}$, and $Z_{22}$ and matrices $\widehat{A}, \widehat{B}$, $\widehat{C}, X_{1}, X_{2}, X_{3}, P_{12}, Q_{12}, R_{1,12}, R_{2,12}$, and $Z_{12}$, such that the following inequalities hold for $\rho_{k}=1$ or $\rho_{k}=0$ :

$$
\left[\begin{array}{ll}
\widetilde{\Omega} & \widehat{\Pi}_{12} \\
* & \widetilde{\Pi}_{22}
\end{array}\right]<0,
$$

where $\widetilde{\Omega}$ and $\widetilde{\Pi}_{22}$ are the same as the corresponding items in (16), $\widehat{\Pi}_{12}$ is derived from $\widetilde{\Pi}_{12}$ in (16) by substituting $\widetilde{\Phi}_{k}$ and $\widetilde{\Gamma}_{k}$ with $\widetilde{\Phi}$ and $\widetilde{\Gamma}$, respectively, and $\widetilde{\Phi}$ and $\widetilde{\Gamma}$ are the same as the corresponding items in (9), then, under the fault detection filter (5) with

$$
\begin{aligned}
& A_{f}=G_{3}^{-T} \widehat{A}^{T} G_{3}^{-1} G_{4}, \\
& B_{f}=G_{3}^{-T} \widehat{B}^{T}, \\
& C_{f}=\widehat{C}^{T} G_{3}^{-1} G_{4},
\end{aligned}
$$

the residual system (9) is asymptotically stable with an $H_{\infty}$ norm bound $\gamma$.

In the following, we will analyze the merits for considering the mutually exclusive distribution characteristic of packet dropouts and network-induced delays.

If the mutually exclusive distribution characteristic of packet dropouts and network-induced delays is neglected, the result in Theorem 2 is described as shown in the following corollary.

Corollary 5. For given scalars $i_{m} \geq 0, i_{M}>0$, and $\gamma>0$, if there exist symmetric positive definite matrices $P_{11}, P_{22}, Q_{11}$, $Q_{22}, R_{1,11}, R_{1,22}, R_{2,11}, R_{2,22}, Z_{11}$, and $Z_{22}$ and matrices $\widehat{A}, \widehat{B}$, $\widehat{C}, X_{1}, X_{2}, X_{3}, P_{12}, Q_{12}, R_{1,12}, R_{2,12}$, and $Z_{12}$, such that

$$
\left[\begin{array}{ll}
\bar{\Omega} & \widetilde{\Pi}_{12} \\
* & \widetilde{\Pi}_{22}
\end{array}\right]<0,
$$

where $\bar{\Omega}$ is derived from $\widetilde{\Omega}$ in (16) by deleting all the items multiplied by $\rho_{k}$ and $\left(1-\rho_{k}\right), \widetilde{\Pi}_{12}$ and $\widetilde{\Pi}_{22}$ are the same as the corresponding items in (16), then, under the fault detection filter (5) with

$$
\begin{gathered}
A_{f}=G_{3}^{-T} \widehat{A}^{T} G_{3}^{-1} G_{4}, \\
B_{f}=G_{3}^{-T} \widehat{B}^{T}, \\
C_{f}=\widehat{C}^{T} G_{3}^{-1} G_{4},
\end{gathered}
$$

the residual system (7) is asymptotically stable with an $H_{\infty}$ norm bound $\gamma$.

The following theorem establishes the relationship between Theorem 2 and Corollary 5.

Theorem 6. Consider the system (7). If the fault detection filter design criterion presented in Corollary 5 is satisfied, then the fault detection filter design criterion in Theorem 2 is also satisfied.

Proof. Define the matrices $\left[\begin{array}{cc}\widetilde{\Omega} & \widetilde{\Pi}_{12} \\ * & \widetilde{\Pi}_{22}\end{array}\right]$ in (16) and $\left[\begin{array}{cc}\bar{\Omega}_{\mathbb{\Pi}_{12}} \\ * & \widetilde{\Pi}_{22}\end{array}\right]$ in (32) as $N$ and $\widetilde{N}$, respectively. Then,

$$
\begin{aligned}
N= & \widetilde{N}-\operatorname{diag}\{0,\left(1-\rho_{k}\right) \widetilde{Q}, \underbrace{0, \ldots, 0}_{6}\} \\
& -\rho_{k} \frac{i_{M}-\bar{i}}{\bar{i}-i_{m}} \Upsilon_{4}^{T} \widetilde{Z} \Upsilon_{4} \\
& -\left(1-\rho_{k}\right) \frac{\bar{i}-i_{m}}{i_{M}-\bar{i}} \Upsilon_{5}^{T} \widetilde{Z} \Upsilon_{5},
\end{aligned}
$$

where $\Upsilon_{4}=\left[\begin{array}{llllllll}0 & I & -I & 0 & 0 & 0 & 0 & 0\end{array}\right]$ and $\Upsilon_{5}=$ $\left[\begin{array}{llllllll}0 & 0 & I & -I & 0 & 0 & 0 & 0\end{array}\right]$. From (34), one can see that if $\widetilde{N}<0$ is satisfied, $N<0$ is also satisfied. This completes the proof.

Remark 7. It has been proved theoretically that the fault detection filter design criterion in Theorem 2 is easier to be satisfied than the fault detection filter design criterion in Corollary 5, which illustrates the merits for considering the mutually exclusive distribution characteristic of packet dropouts and network-induced delays. Notice that the mutually exclusive distribution characteristic of packet dropouts and network-induced delays is adopted to deal with bounding inequalities for products of vectors. Even for the systems in $[30,31]$ without considering the occurrence of faults, adopting the mutually exclusive distribution characteristic of packet dropouts and network-induced delays will also introduce better results. The corresponding results are omitted here for briefness.

In the following, we will show the effectiveness of the proposed fault detection filter design by the simulation results. 


\section{Simulation Results and Discussion}

To illustrate the effectiveness of the proposed fault detection filter design, we consider the following linear model for the motion of ships, see also [32-34] for similar models:

$$
\begin{aligned}
& \dot{x}(t)=A x(t)+B u(t)+B_{\omega} \omega(t)+E_{f} f(t), \\
& y(t)=C x(t),
\end{aligned}
$$

where $x(t)=\left[\begin{array}{llll}z_{\delta}(t) & \dot{z}_{\delta}(t) & \theta_{\delta}(t) & \dot{\theta}_{\delta}(t)\end{array}\right]^{T}$ is the state vector, $u(t)=\left[\begin{array}{ll}a_{1 \delta}(t) & a_{2 \delta}(t)\end{array}\right]^{T}$ is the control input vector, $\omega(t)=$ $\left[\omega_{1}^{\prime}(t) \omega_{2}^{\prime}(t)\right]^{T}$ is the unknown disturbance input, and $f(t)$ is the fault signal. The physical meaning for $z_{\delta}(t), \dot{z}_{\delta}(t), \theta_{\delta}(t)$, $\dot{\theta}_{\delta}(t), a_{1 \delta}(t)$, and $a_{2 \delta}(t)$ can be found in [34] and the references therein. with

The system matrices in (35) are similar to the ones in [34]

$$
\begin{aligned}
& A=\left[\begin{array}{cccc}
0 & 1 & 0 & 0 \\
-0.1923 & -14.3338 & -210.7117 & -87.9924 \\
0 & 0 & 0 & 1 \\
-0.0002 & -0.015 & -0.2210 & -9.9805
\end{array}\right] \text {, } \\
& B=\left[\begin{array}{cc}
0 & 0 \\
-30.0785 & -37.0679 \\
0 & 0 \\
1.3379 & -2.3439
\end{array}\right] \\
& B_{w}=\left[\begin{array}{cc}
0 & 0 \\
18.7186 & -0.0636 \\
0 & 0 \\
0.5736 & -0.0006
\end{array}\right] \text {, } \\
& E_{f}=-B \times\left[\begin{array}{l}
1 \\
0
\end{array}\right], \quad C=\left[\begin{array}{llll}
0 & 1 & 0 & 1
\end{array}\right] .
\end{aligned}
$$

The parameters for the fault weighting system (6) are chosen as $A_{W}=0.8, B_{W}=0.2, C_{W}=0.9$, and $D_{W}=-0.6$. The active-varying sampling periods are considered in this paper, and suppose that the sampling periods switch in the finite set $\theta=\{0.05 \mathrm{~s}, 0.1 \mathrm{~s}\}, i_{m}=1, i_{M}=3$. Then one has $\bar{i}=2$. Define $I_{2}=\left[\begin{array}{lllll}0 & 1 & 0 & 0 & 0\end{array}\right]^{T}$ and $I_{4}=\left[\begin{array}{lllll}0 & 0 & 0 & 1 & 0\end{array}\right]^{T}$. To avoid that some elements of the obtained matrix $B_{f}$ are close to zero, we assume that $\widehat{B} I_{2}+I_{2}^{T} \widehat{B}^{T}+0.1<0, \widehat{B} I_{4}+I_{4}^{T} \widehat{B}^{T}+0.1<$ 0 . Discretizing the system (35), constructing the closed-loop system, and solving the fault detection filter design criterion presented in Theorem 2, one has

$$
A_{f}=\left[\begin{array}{ccccc}
0.7845 & 0.0189 & -0.1688 & 0.0102 & -0.0001 \\
0.0033 & 0.3841 & 0.0737 & 0.0450 & -0.0001 \\
-0.0307 & 0.0408 & 0.4627 & 0.0350 & 0.0007 \\
0.0036 & 0.0472 & 0.0351 & 0.9927 & -0.0001 \\
0.0001 & -0.0001 & 0.0009 & -0.0000 & 1.0000
\end{array}\right],
$$

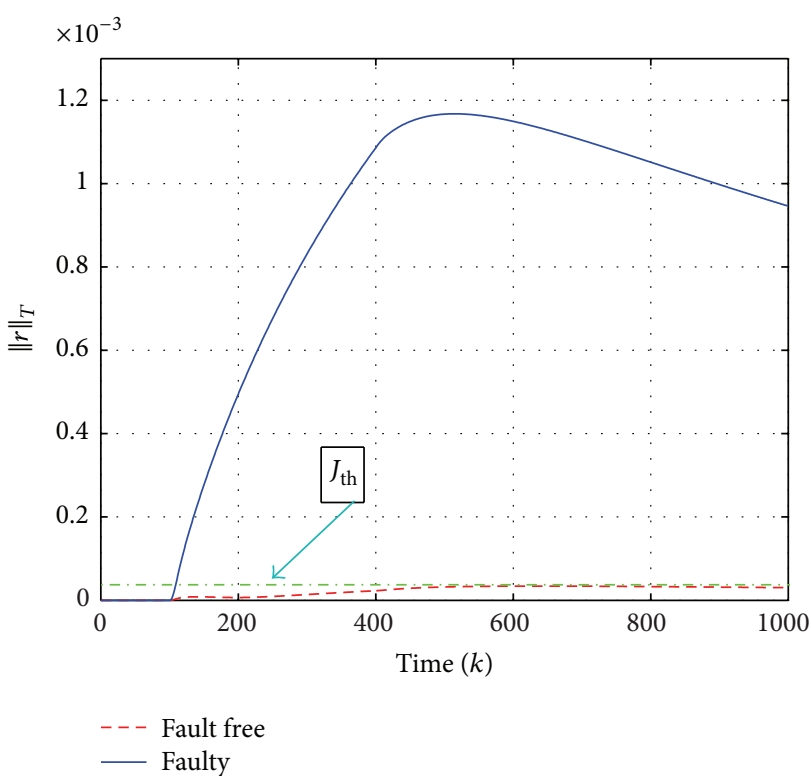

FIGURE 1: Residual evaluation function response $\|r\|_{T}$ for $h_{k}=h_{1}$.

$$
\begin{aligned}
B_{f} & =\left[\begin{array}{c}
0.0156 \\
0.0233 \\
0.0045 \\
-0.0023 \\
-0.0000
\end{array}\right], \\
C_{f} & =\left[\begin{array}{lllll}
-0.0127 & 0.0020 & 0.0170 & -0.0035 & -0.0002
\end{array}\right] .
\end{aligned}
$$

Suppose that the initial state of the augmented closed-loop system (7) is $\xi_{0}=\left[\begin{array}{llllllllll}0 & 0 & 0 & 0 & 0 & 0 & 0 & 0 & 0 & 0\end{array}\right]^{T}$. For $0 \leq k<$ $1000, i_{k}$ will switch between 1 and 2 in cycles. For $0 \leq k<99$ and $402 \leq k<1000, u_{k}, \omega_{k}$, and $f_{k}$ are assumed to be zero. For $100 \leq k<402$, suppose that $u_{k}=\left[\begin{array}{ll}0.1 & 0.1\end{array}\right]^{T}$, $\omega_{k}=\left[\begin{array}{ll}0.1 \sin (k) & 0.1 \sin (k)\end{array}\right]^{T}$, and in the case that a fault occurs, suppose that $f_{k}=2$, where $u_{k}, \omega_{k}$, and $f_{k}$ denote the values of $u(t), \omega(t)$, and $f(t)$ at the $k$ th sampling instant, respectively.

It should be pointed out that the fault detection filter design method in Theorem 2 is applicable for $h_{k}=h_{1}$, switched sampling periods (i.e., $h_{k}$ switches between $h_{1}$ and $h_{2}$ in cycles), and $h_{k}=h_{2}$. The residual evaluation function response $\|r\|_{T}$ for $h_{k}=h_{1}$, switched sampling periods, and $h_{k}=h_{2}$ is presented in Figures 1, 2, and 3, respectively. Considering the fault detection logic presented in (12) and from Figures 1, 2, and 3, one can see that the newly proposed fault detection scheme can not only reflect the occurrence of faults in time but also recognize the faults without confusing faults with the disturbance $\omega_{k}$.

Define $n$ as the number of needed sampling periods for fault detection. Table 1 shows the numbers of needed sampling periods for fault detection corresponding to different 


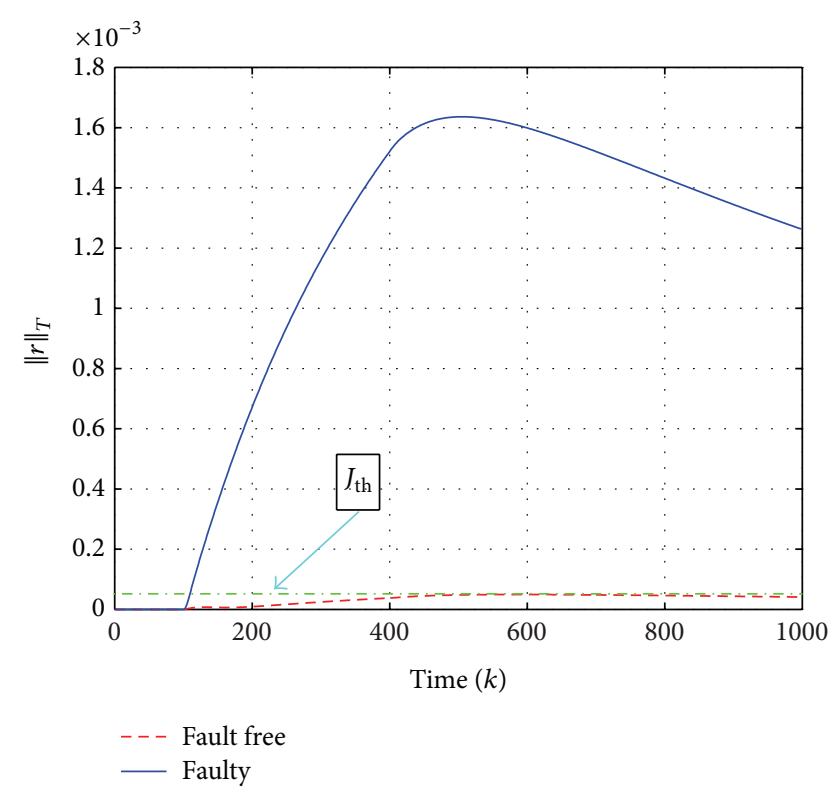

Figure 2: Residual evaluation function response $\|r\|_{T}$ for switched sampling periods.

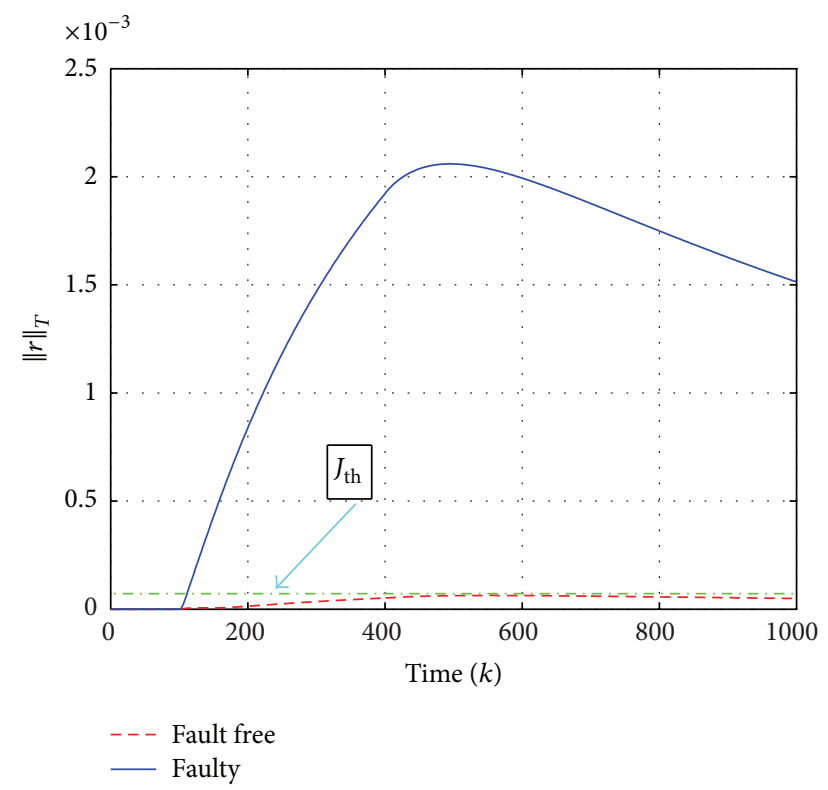

FIgURE 3: Residual evaluation function response $\|r\|_{T}$ for $h_{k}=h_{2}$.

cases. As one can see in Table 1 , the shorter the sampling period, the smaller the number of needed sampling periods for fault detection, which illustrates the effectiveness of the newly proposed active-varying sampling-based fault detection scheme.

\section{Conclusions}

The fault detection filter design for continuous-time NCSs considering packet dropouts and network-induced delays
TABLE 1: The numbers of the needed sampling periods for fault detection.

\begin{tabular}{lccc}
\hline Method & $h_{k}=h_{1}$ & $h_{k}=h_{1}$ or $h_{k}=h_{2}$ & $h_{k}=h_{2}$ \\
\hline$n$ & 10 & 11 & 12 \\
\hline
\end{tabular}

has been studied in this paper. The active-varying sampling period method has been introduced to establish a new discretized model and improve the fault detection performance for the considered NCSs. The fault detection filter design criterion which considers the mutually exclusive distribution characteristic of packet dropouts and networkinduced delays has been proved theoretically to be less conservative than the criterion without considering such a mutually exclusive distribution characteristic. The merits and effectiveness of the proposed active-varying samplingbased fault detection filter design have been verified by the simulation results.

\section{Conflict of Interests}

The authors declare that there is no conflict of interests regarding the publication of this paper.

\section{Acknowledgments}

This work was supported in part by the National Science Foundation of China (Grant no. 61004025, Grant no. 61210306066, Grant no. 61374063, Grant no. 61104106, and Grant no. 61104029), the "333 Project" in Jiangsu Province, China, the "Qing Lan Project" in Jiangsu Province, China, the Key Project for International Science and Technology Cooperation and Inviting Experts in Universities of Jiangsu Province, China, the Natural Science Foundation of Liaoning Province, China (Grant no. 201202156), and the Program for Liaoning Excellent Talents in University (LNET), China (Grant no. LJQ2012100).

\section{References}

[1] D. Wang, J. Wang, and W. Wang, " $\mathrm{H}_{\infty}$ controller design of networked control systems with markov packet dropouts," IEEE Transactions on Systems, Man, and Cybernetics: Systems, vol. 43, no. 3, pp. 689-697, 2013.

[2] H. Yang, Y. Chen, and T. Ren, " $\mathrm{H}_{\infty}$ filtering for networked systems with bounded measurement missing," Mathematical Problems in Engineering, vol. 2014, Article ID 375793, 10 pages, 2014.

[3] B. Addad, S. Amari, and J.-J. Lesage, "A virtual-queuing-based algorithm for delay evaluation in networked control systems," IEEE Transactions on Industrial Electronics, vol. 58, no. 9, pp. 4471-4479, 2011.

[4] D. Zhang, Q.-G. Wang, L. Yu, and Q.-K. Shao, " $\mathrm{H}_{\infty}$ filtering for networked systems with multiple time-varying transmissions and random packet dropouts," IEEE Transactions on Industrial Informatics, vol. 9, no. 3, pp. 1705-1716, 2013. 
[5] F.-L. Qu, Z.-H. Guan, T. Li, and F.-S. Yuan, "Stabilisation of wireless networked control systems with packet loss," IET Control Theory \& Applications, vol. 6, no. 15, pp. 2362-2366, 2012.

[6] C. Peng and Y. Tian, "Delay-dependent robust $H \infty$ control for uncertain systems with time-varying delay," Information Sciences, vol. 179, no. 18, pp. 3187-3197, 2009.

[7] Y. Wang, X. Zhou, and X. Zhang, "Hळ filtering for discretetime genetic regulatory networks with random delay described by a Markovian chain," Abstract and Applied Analysis, vol. 2014, Article ID 257971, 12 pages, 2014.

[8] S. Hu, X. Yin, Y. Zhang, and E. G. Tian, "Event-triggered guaranteed cost control for uncertain discrete-time networked control systems with time-varying transmission delays," IET Control Theory \& Applications, vol. 6, no. 18, pp. 2793-2804, 2012.

[9] C. Peng, Q.-L. Han, and D. Yue, "To transmit or not to transmit: a discrete event-triggered communication scheme for networked takagi-sugeno fuzzy systems," IEEE Transactions on Fuzzy Systems, vol. 21, no. 1, pp. 164-170, 2013.

[10] C. Luo, X. Wang, and Y. Ren, "Consensus problems in multiagent systems with event-triggered dynamic quantizers," Mathematical Problems in Engineering, vol. 2014, Article ID 162658, 8 pages, 2014.

[11] Z.-H. Pang and G.-P. Liu, "Design and implementation of secure networked predictive control systems under deception attacks," IEEE Transactions on Control Systems Technology, vol. 20, no. 5, pp. 1334-1342, 2012.

[12] J. Zhang, Y. Xia, and P. Shi, "Design and stability analysis of networked predictive control systems," IEEE Transactions on Control Systems Technology, vol. 21, no. 4, pp. 1495-1501, 2013.

[13] H. Dong, Z. Wang, D. W. C. Ho, and H. Gao, "Variance-constrained $H \infty$ filtering for a class of nonlinear time-varying systems with multiple missing measurements: the finite-horizon case," IEEE Transactions on Signal Processing, vol. 58, no. 5, pp. 2534-2543, 2010.

[14] J. Hu, Z. Wang, B. Shen, and H. Gao, "Quantised recursive filtering for a class of nonlinear systems with multiplicative noises and missing measurements," International Journal of Control, vol. 86, no. 4, pp. 650-663, 2013.

[15] G. Guo and H. Jin, "A switching system approach to actuator assignment with limited channels," International Journal of Robust and Nonlinear Control, vol. 20, no. 12, pp. 1407-1426, 2010.

[16] Y. Niu, T. Jia, X. Wang, and F. Yang, "Output-feedback control design for NCSs subject to quantization and dropout," Information Sciences, vol. 179, no. 21, pp. 3804-3813, 2009.

[17] C. Peng, Y.-C. Tian, and D. Yue, "Output feedback control of discrete-time systems in networked environments," IEEE Transactions on Systems, Man, and Cybernetics A: Systems and Humans, vol. 41, no. 1, pp. 185-190, 2011.

[18] D. Wang, J. Wang, and W. Wang, "Output feedback control of networked control systems with packet dropouts in both channels," Information Sciences, vol. 221, pp. 544-554, 2013.

[19] D. Yue, E. Tian, Z. Wang, and J. Lam, "Stabilization of systems with probabilistic interval input delays and its applications to networked control systems," IEEE Transactions on Systems, Man, and Cybernetics A: Systems and Humans, vol. 39, no. 4, pp. 939-945, 2009.

[20] R. Lozano, P. Castillo, P. Garcia, and A. Dzul, "Robust prediction-based control for unstable delay systems: application to the yaw control of a mini-helicopter," Automatica, vol. 40, no. 4, pp. 603-612, 2004

[21] H. Gao, J. Wu, and P. Shi, "Robust sampled-data $H \infty$ control with stochastic sampling," Automatica, vol. 45, no. 7, pp. 17291736, 2009.

[22] Y.-L. Wang and G.-H. Yang, " $\mathrm{H}_{\infty}$ controller design for networked control systems via active-varying sampling period method," Acta Automatica Sinica, vol. 34, no. 7, pp. 814-818, 2008.

[23] Y. Wang, S. X. Ding, H. Ye, L. Wei, P. Zhang, and G. Wang, "Fault detection of networked control systems with packet based periodic communication," International Journal of Adaptive Control and Signal Processing, vol. 23, no. 8, pp. 682-698, 2009.

[24] X. He, Z. Wang, Y. D. Ji, and D. H. Zhou, "Robust fault detection for networked systems with distributed sensors," IEEE Transactions on Aerospace and Electronic Systems, vol. 47, no. 1, pp. 166-177, 2011.

[25] D. Huang and S. K. Nguang, "Robust fault estimator design for uncertain networked control systems with random time delays: an ILMI approach," Information Sciences, vol. 180, no. 3, pp. 465-480, 2010.

[26] B. Liu and Y. Xia, "Fault detection and compensation for linear systems over networks with random delays and clock asynchronism," IEEE Transactions on Industrial Electronics, vol. 58, no. 9, pp. 4396-4406, 2011.

[27] H. Dong, Z. Wang, J. Lam, and H. Gao, "Fuzzy-model-based robust fault detection with stochastic mixed time delays and successive packet dropouts," IEEE Transactions on Systems, Man, and Cybernetics B: Cybernetics, vol. 42, no. 2, pp. 365-376, 2012.

[28] Y. Zhao, J. Lam, and H. Gao, "Fault detection for fuzzy systems with intermittent measurements," IEEE Transactions on Fuzzy Systems, vol. 17, no. 2, pp. 398-410, 2009.

[29] K. Gu, V. L. Kharitonov, and J. Chen, Stability of Time Delay Systems, Birkhäuser, Boston, Mass, USA, 2003.

[30] X. Jia, D. Zhang, X. Hao, and N. Zheng, "Fuzzy $H_{\infty}$ tracking control for nonlinear networked control systems in T-S fuzzy model," IEEE Transactions on Systems, Man, and Cybernetics B: Cybernetics, vol. 39, no. 4, pp. 1073-1079, 2009.

[31] X. Jiang, Q.-L. Han, S. Liu, and A. Xue, "A new $\mathrm{H}_{\infty}$ stabilization criterion for networked control systems," IEEE Transactions on Automatic Control, vol. 53, no. 4, pp. 1025-1032, 2008.

[32] T. I. Fossen, Guidance and Control of Ocean Vehicles, Wiley, Chichester, UK, 1994.

[33] J. van Amerongen, P. G. M. van der Klugt, and H. R. van Nauta Lemke, "Rudder roll stabilization for ships," Automatica, vol. 26, no. 4, pp. 679-690, 1990.

[34] J. S. Ren and Y. S. Yang, "Controller design of hydrofoil catamaran with dynamical output-feedback $\mathrm{H}_{\infty}$ scheme," Journal of Traffic and Transportation Engineering, vol. 5, no. 1, pp. 45-48, 2005 (Chinese). 


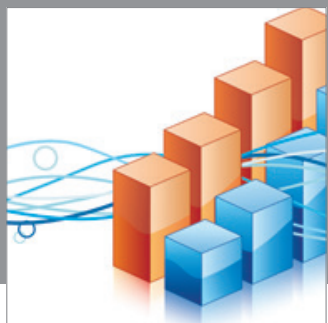

Advances in

Operations Research

mansans

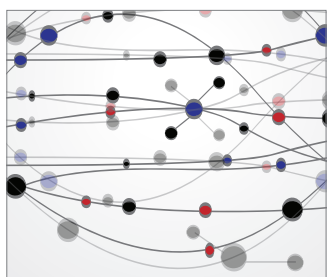

The Scientific World Journal
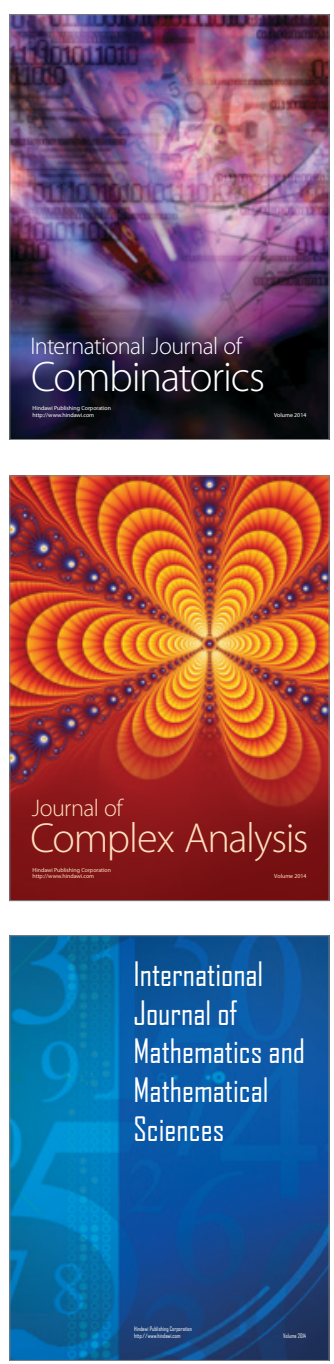
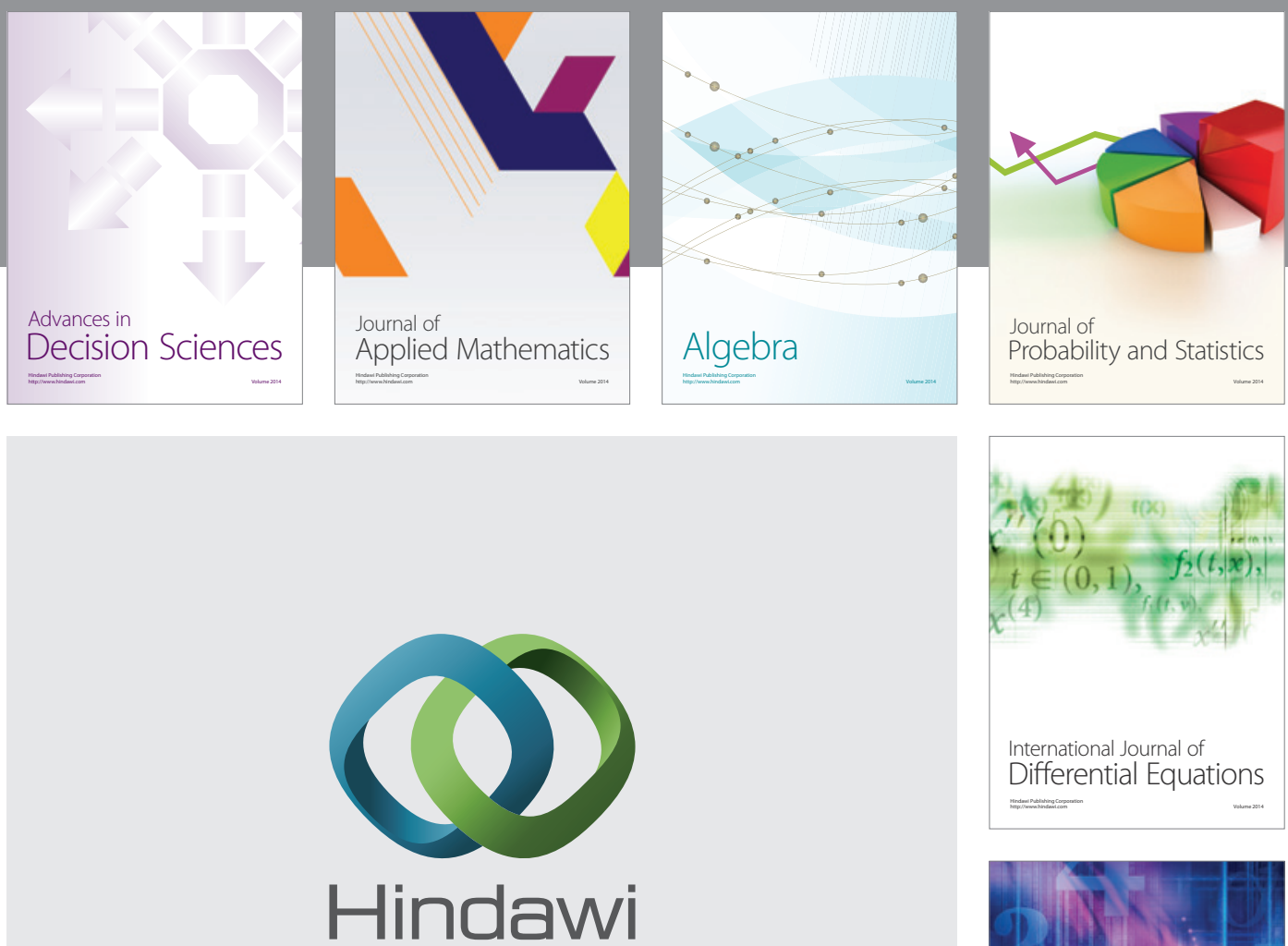

Submit your manuscripts at http://www.hindawi.com
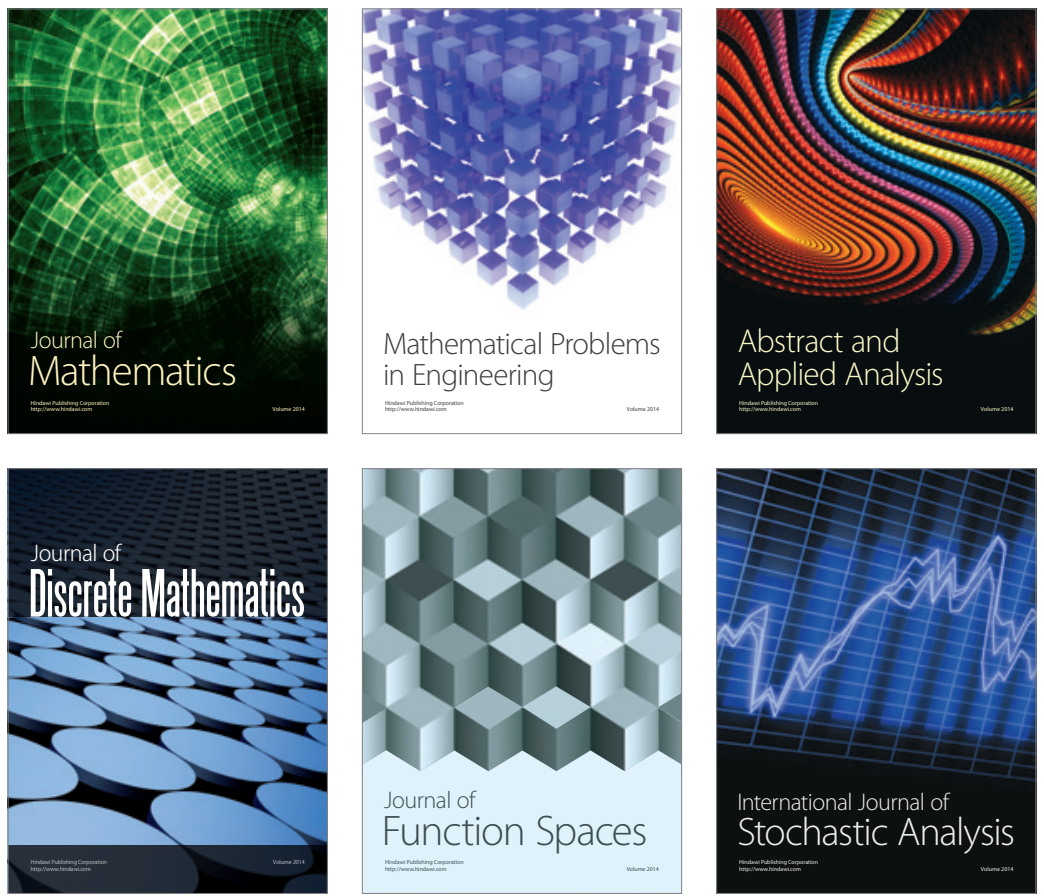

Journal of

Function Spaces

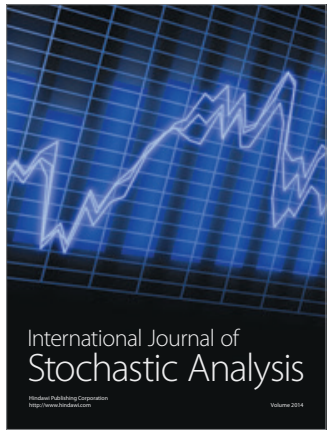

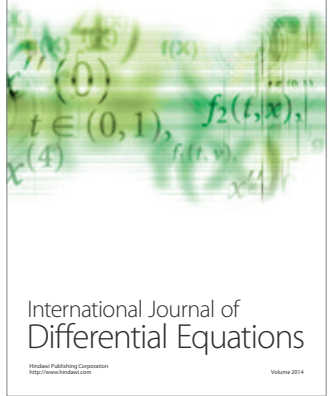
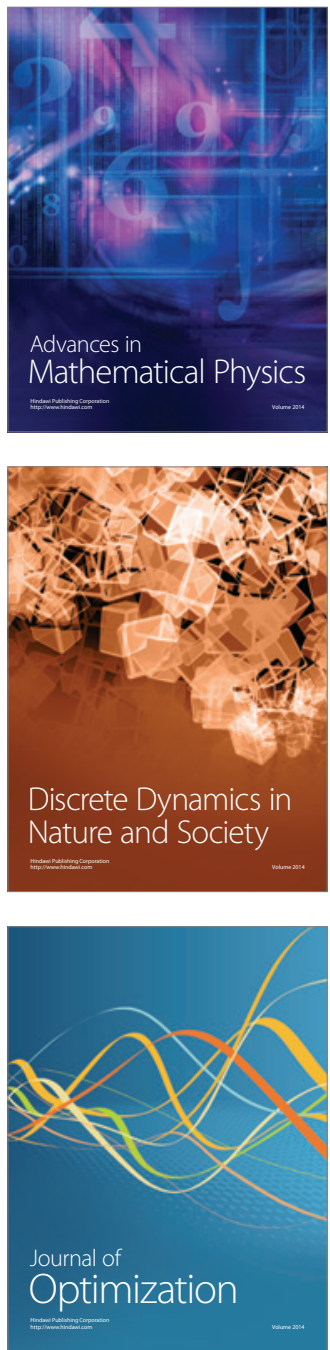